\title{
Vehicle Maintenance Prediction, Lane and Drowsiness Detection using Machine Learning
}

\author{
Sanath T.S. \\ Undergraduate Researcher
}

\begin{abstract}
This research paper includes about the driver's safety. It has three different models. They are Drowsiness detection system, Lane detection system and Vehicle maintenance system. Drowsiness is one of the major reasons for road accidents. To overcome this, prediction of drowsiness is developed. In fact, the drowsiness affects the performance, capability, physiological indices. These parameters provide information about driver's state. First, the camera captures the driver's face and then drowsy state is recognized by the machine learning algorithms. As a result, an alert can be initiated. Second, while lane changing one has to be very conscious, lane changing is one of the major cause of accidents. Computer vision and machine learning will be used to understand the change of lane, any changes in the path will be notified to the driver and an alert will be initiated. In the paper, we are using canny edge detection, gaussian smoothing and hogs transform which will increase the efficiency of the system as a result we can avoid the road accidents and ensure the safety. Third model in this paper is vehicle maintenance system. Here, based on the previous maintenance records, date and time of last service, the system will predict the next date of maintenance. We are classifying the model using Sklearn package and support vector machine algorithm. The generated model can predict and perform at least an accuracy of $90 \%$ and can be improvised by using the large datasets.
\end{abstract}

\section{Keywords}

Machine learning, drowsiness detection, algorithms, canny edge detection, hogs transform, gaussian smoothing, computer vision, classification, Sklearn tools, and support vector machine (SVM).

\section{INTRODUCTION}

Every human being needs sleep, lack of sleep cause human inactive, improper reflex, losing focus, gets deviated which decreases the capability to make proper decisions which is necessary for driving a vehicle. As per WHO records about 1.25 million of people were injured or dead due to accidents in a year. Some of them neglect the traffic rules, like driving the vehicle with over speed, crossing the signals, crossing the lane, also having technical issues with the break's failure, tires. To mitigate these issues this paper focuses on the solution to reduce the fatal cases by providing a smart drowsiness detection system. This model has an accuracy of $90 \%$. Machine learning, Computer vision are being used in this model which are the subset of AI and it allows the user to train the system and predict the output in a certain range. This technology helps to reduce the gap between human and machines. Computer vision is the library which captures, understand the images to perform image processing. It helps in processing and extraction of the data to generate the information and also it use's other libraries which play an important role in the system. It has a graphical user interface (GUI) which make the operating systems easy for users to use. Where you can simply create own windows for generating output of certain model. We have 3 models: drowsiness detection, lane detection and vehicle maintenance prediction. The outcome of this model is to alert and predict whenever the driver is drowsy, changing his track and when vehicle is not maintained well. The aim of this paper is to implement this system in the vehicle, and ensure driver's safety. Algorithms are used to process the signals and to give relevant output.

\section{RELATED WORKS}

Wire Willie developed drowsiness detection algorithm named PERCLOS. The feature of this algorithm is it calculates the values of eyes closed over a period of time. PERCLOS has the accuracy of $80-90 \%$.

Random forest model created by MC Donald using the angle of steering to detect drowsiness. The random forest models' algorithms have higher efficiency than the PERCLOS model.

Similarly, MC Donald created a hidden Markova model to find whether the driver is drowsy or not in a time series data. MC Donald found that by using hidden marcov model the efficiency of the model increased and reduced the false detection.

The system developed by Mobilise, detects the lane marking on the road and alerts when the lane crosses over the actual lane without any manual movement. Nowadays auto mobile companies are integrating this model to maintain driver's safety.

A lane departure system was introduced by the general motors in 2008, on Cadillac model and Buick lucerne model. To generate an audible tone on the display of ECU in the vehicle, many automobile companies have taken part in this by adding lane departure warning in their systems. Like BMW, Mercedes and AUDI.

\section{DATASET}

Dataset is a collection of data in a tabular form or images. Machine learning is completely reliable on the data, the more we give the data the system becomes more intelligent. It can use any algorithm for training the model, having the enough data can give more training and accuracy to the system.

In this paper we have three different data sets to train every model. The first model is drowsiness detection system, here the images are taken from Kaggle. Kaggle is platform which provides dataset for every topic. The collection of images for this model is of two types, images with eyes open and images with eyes closed. 


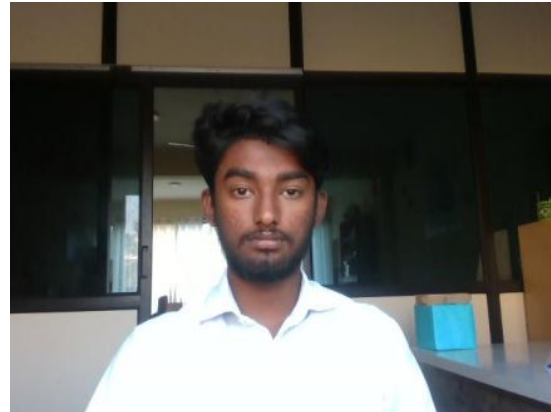

Figure (1): Image from dataset

In the second model, the lane detection system detects the lane and alerts the person. Here, the dataset contains the images with the lanes highlighted. Images with normal lanes can also train the model using video of the car moving in particular lane which makes the system more accurate. In this model images are presented in a folder for different categories which are considered as the dataset of the model.

Similarly, the third model also require dataset for training but the difference between this model and other two models is the first two models have image dataset whereas the third model has tabular dataset which is a CSV dataset. In this dataset there are six columns they are month, date, year, engine, breaks, output and these are the parameters which help in achieving the required output. This data set is not existing, it was created to execute the operation. Here every parameter has different values which are dependent on each other directly or indirectly, which states that any change in the parameter will cause a drastic change in dataset.

Hence, dataset is necessary for training the model and to achieve accuracy, efficiency, reliability and many more which increases the systems capability. Here the datasets are created to train our model and make it accurate.

\section{ALGORITHM}

Algorithm is an instruction used for computer to perform a specific task in a particular order.

Algorithms used in this system are:

\subsection{Support Vector Machine (SVM)}

The aim of support vector machine is to find the hyperplane in a dimensional space for classifying a data given to the model. There are many hyperplanes that can be selected. Support vector machine also used to find a hyperplane which has maximum margin between the given data points. Actually, the hyperplane is a boundary which classify the data points. The data points in both sides of the plane is denoted as the different classes and hyperplane dimensions are dependent on the features of the model. If our model has the feature of 2 then our hyperplane will be 2D. If feature value crosses 3 then it is difficult for SVM to classify. For building SVM model it requires few parameters like large margin intuition, cost function, gradients and many more.

(Gandhi, 2018). Support vector machine can be used as regression model or classification model, currently in this system SVM model is used as classification model because SVM produces higher accuracy for the system which make system more efficient. As already discussed above classification is done on hyperplane that differentiate the two parameters which are available in the dataset. Let's move deep into it and get to know how does the classification goes on. The model creates few hyperplanes which are dividing the parameters in a certain manner, then the model starts filtering the hyperplane by considering margin of each hyperplane. The distance between datapoints and hyperplanes gives an accurate plane which divides the dataset set accurately. Margin is the distance between the dataset and hyperplane.

\subsection{Eye Blink Algorithm}

The blink of eye can be detected by considering facial landmarks, most of the libraries provide features of faces with in the interested region. Example dlib is the library which provides trained model were the output is to plot coordinates on the considered region of image and also plot 68 points on the input image. The system considers the 37-46 coordinates which are related to eye. (Rosebrock, 2017). The main import factor is eye aspect ratio which is used to help the system to identify the blink of eye.

The eye aspect ratio remains constant than the eye is considered as open and when eye aspect ratio is zero than eye is considered as closed. As the face detection results the bounding boxes on the face similarly the bonding boxes are also created on eyes and face which gives a clarity about the point of interest.

\subsection{Canny Edge Detection:}

Canny edge detection is type of edge detection which deals with multistage algorithms for wide edges in image. John F. Canny developed this canny edge detection in 1986. (R. Fisher, 2003), This detection uses filter based on gaussian derivates to compute the gradient intensity. The gaussian is used to reduce the noise in the image, one edge pixel is thinned down from the image by eliminate non maximum pixel in gradient magnitude and finally the edge pixel is represented on the gradient magnitude. This technique can be modified by 3 parameters. They are noise, width and threshold values in image. If we have low error rate in detection that indicates the system has got edges accurately.

\subsection{Gaussian Smoothing}

(S. Perkins, 2003), The gaussian smoothing is method works as convolutional operator which used for blurring image, smooth the image, and remove noise from the image. Similarly, as the filter operation but the advantage is Gaussian smoothing uses different kernel which denotes the shape of the gaussian function.

\subsection{Hough Transform}

(R. Fisher, 2003), Hough transform is used for detecting lines and also other shapes(or)curves. It is developed by Hough in 1960 and used for detecting lines in the image. The objective of using Hough transform is to find the lines in an image or video, we can detect lines(or)curves when we have particular transform. Theoretically, it can be solved by correlation and linear elements structures.

Hough transform is a method which is used for image analysis and image processing. The previous model of Hough transform was only used for detecting lines in the image (or) video. As the technology increased the algorithm got efficient that it can detect shapes like circles and ellipse. For analyzing the digital images different geometric shapes were creating problems. For training the data image is converted into edge detector image which causes missing of pixels at certain area. To overcome this problem Hough transform is introduced.

\section{METHODOLOGY (OR) DATA PROCESSING}

Let's discuss about the first model: Drowsiness detection system. This system detects weather driver is drowsy or not by considering eyes and facial landmarks. Flow chart is shown in 
Fig (2) the first step is to perform the face recognition in order to detect blink of an eye, where the system recognizing the driver eyes and the calculate the speed of eye blink and provide an appropriate output.

HAAR cascade method which uses OpenCV to detect the human faces. Human eye is dark in color and nose is bright in color. Hence HAAR cascade technique is used for extracting the face information using OpenCV. This method recognizes the position of the eye on human face. In the system the face is monitored continuously till he/she stops the functioning of it. The mean shift method is used for finding the center of image for image distribution, where the data is contributed on a plane. For achieving the dense part of the image, we must obtain position and radius of the image. Eye detection, Dlib is the open-source library used for detecting the blink and also calculating the eye aspect ratio were the eye aspect ratio has the threshold of 0.3 if the threshold is constant than system consider as the eyes are open, if the threshold value is decreased from 0.3 than system will consider as the drowsy state. Dlib creates the bounding boxes on eye which indicates the improvement performance of the system.

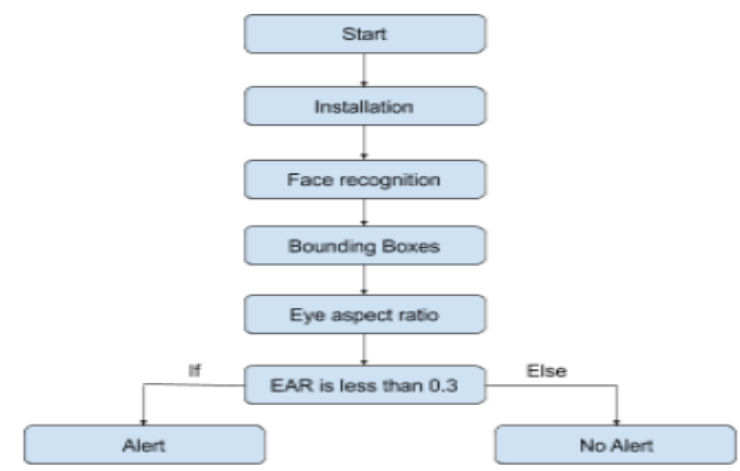

Fig(2):Flowchart of drowsiness detection system

The second model which is lane detection, there are steps to be followed for this model which are given below in the flowchart Fig(3)

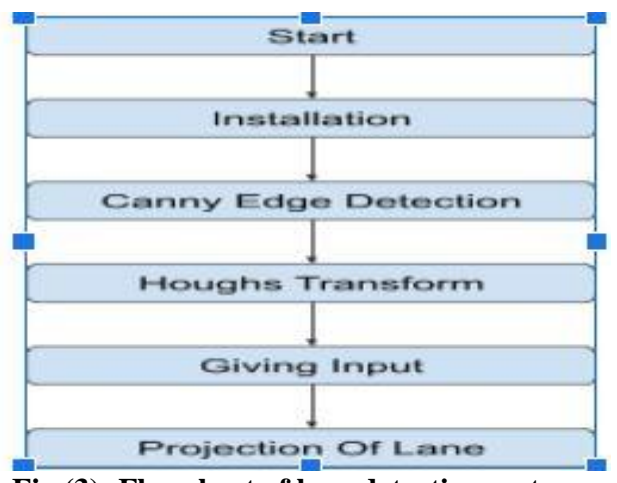

Fig (3): Flowchart of lane detection system

Hough transform is method used for extracting the features that can be utilized for the analysis of the image and image processing, (Rashmi N. Mahajan \& Dr. A. M. Patil, 2015). As already discussed Hough transform was only used for the detection of lines in the image and video, which represents Hough transform, it also detects the shape like circle, ellipse etc. In this model the edge detector is used for achieving the points on the image but by using edge detector the pixels were missing from the image which caused a problem. For resolving this problem Hough transform is considered. Hough transform is the efficient method to detect line in any conditions like noise etc. By creating equation for every possible line in the image and all the pixels gained in edge detection are grouped in a single class. The image is converted into binary image by threshold of some value for detecting the lines in image. It considers two parameters $\mathrm{x}$ and $\mathrm{y}$ were the $\mathrm{y}$ is angle and $\mathrm{x}$ is distance, every line has its own Hough transform and also has discrete set of angles. An accumulator, which is an array used for detection of line, the value of accumulator is equal transforms parameters so, the predominant lane is selected by the parameter having highest vote present on image plane. Usually, Hough transform is used for detecting the straight line when the system gets improved it can also be used for detecting the curves.

Edge detection is a method used for extracting an image or identifying the points on the image which differ in shade sharply. Edge detection is also used for processing images for feature detection and extraction. By this algorithm we can reduce the data and also remove the less amount of information in image, if this method is achieved successfully then interpretation of image becomes easy and original data will be simplified. But it's not possible to achieve this outcome by an ideal edge detection. So we use a detection algorithm called canny edge detection which detects the edges in image, this technique is used for the multistage algorithms and the objective of using this method to achieve optimal edge detection.

The main task is to project boundaries on the lane of image plane or video plane, by using Hough transform and edge detection line, curves present on the image are detected. By using gradient direction features, line function, line prior function, the lane probability is achieved by these parameters by using lane probability. The geometry structured is designed by the system to project the trajectory on the line detected by the algorithm.

The last model in the system is vehicle maintenance system, this model is classification model, where we are using the support vector machine for classifying the dataset. The library named pandas reads the dataset, further the dataset is divided into training and testing were training is $80 \%$ and testing is $20 \%$ After splitting the datasets the model is imported which is support vector machine than the dataset is fitted into the model and then the model is trained and system will become capable of predicting after the training. For integrating these three models in a system button condition are used. To represent the system graphical user interface is added which makes the user to understand the operation of the system easily.

\section{RESULTS}

In this paper the system has three different models, when you run the program the system will direct you to graphical user interface (GUI), where the window consists of three buttons. The first button named as drowsiness detection, second button as lane detection and third button named as vehicle maintenance.

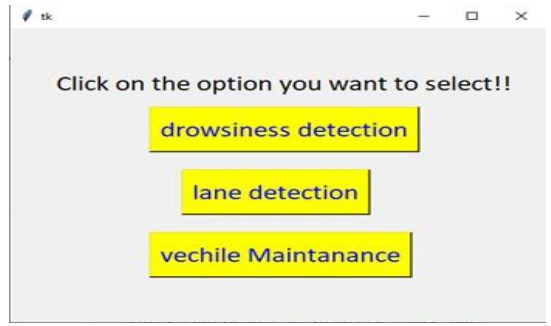

Fig (4): Image of main GUI 
When you select the first button it will redirect you to the drowsiness detection models. GUI consists of the live feed given by the camera with the bounding boxes on face and eyes and when the driver closes his eye for more than $6-7$ seconds than system will give the alert stating driver is drowsy.

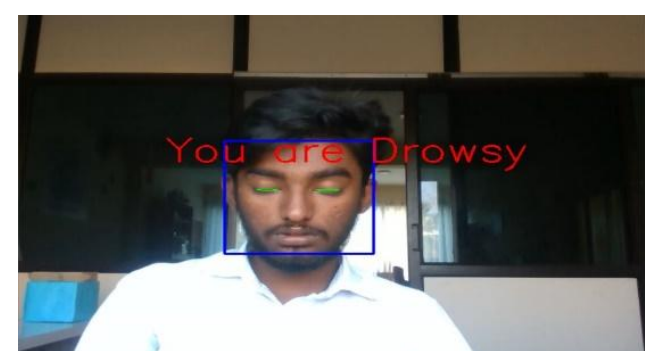

Fig (5): Output of drowsiness detection system

If you select the button number two then it will direct you to the lane detection GUI. The window will ask the video link for detecting the lane in the video, then the system will generate the boundaries on the video link the user has provided.

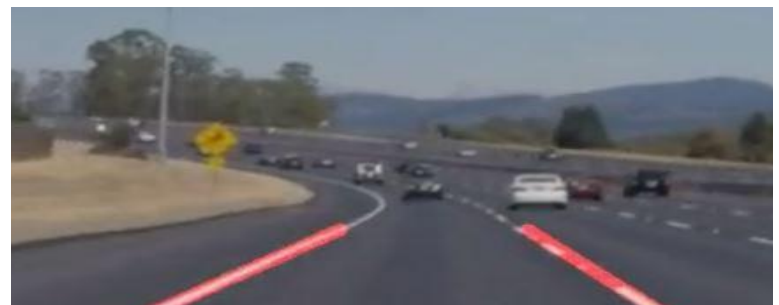

Fig(6):Result of lane detection system

If the user selects the third button then the system will direct you to the vehicle maintenance systems GUI. In this GUI the system asks for a few inputs from the user like date, day and month of last servicing, any issue with the engine and breaks. When we submit the value, the model will predict the output that vehicle needs servicing in 10 days or more.

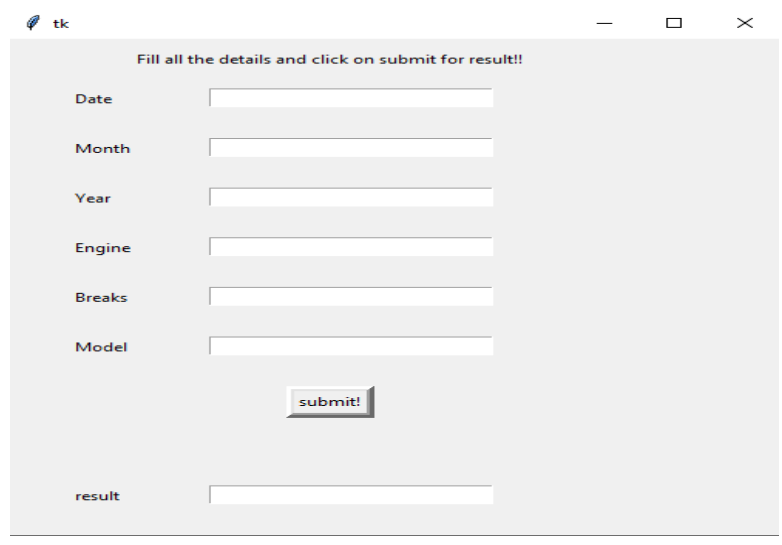

Fig (7): GUI of vehicle maintenance prediction

Table (1): Table for output accuracy

\begin{tabular}{|c|c|c|}
\hline System & Output & Accuracy \\
\hline $\begin{array}{c}\text { Drowsiness } \\
\text { detection } \\
\text { system }\end{array}$ & $\begin{array}{c}\text { Detect weather the } \\
\text { driver is drowsy or not. }\end{array}$ & $90 \%$ \\
\hline $\begin{array}{c}\text { Lane detection } \\
\text { system }\end{array}$ & $\begin{array}{c}\text { Detect weather vehicle } \\
\text { moving in proper lane }\end{array}$ & $93 \%$ \\
\hline
\end{tabular}

\begin{tabular}{|c|c|c|}
$\begin{array}{c}\text { Maintenance } \\
\text { prediction } \\
\text { system }\end{array}$ & $\begin{array}{c}\text { Predicts when vehicles } \\
\text { should be given to } \\
\text { servicing }\end{array}$ & \\
\hline
\end{tabular}

\section{FUTURE SCOPE}

Make the model more accurate and efficient, continuous monitoring of the driver whether he/she is drowsy and giving feedback to their situation and take action. Currently these systems have no zoom adjustment while performing operation. In future, we can look forward towards cameras for capturing only eyes. We can add a few other systems to the vehicle to make the driver and the passenger safe like a seat belt detection system, which detects whether the person is wearing a seatbelt or not, detecting the moving vehicles which is used to identify the position of the vehicles in the same lane etc. You can also modify our lane detection system such that it can say which lane is the safest lane to move by considering the surrounding and most important future works which will give suggestions to measure and maintain the vehicle in the proper way without taking any parameters as input. There are many future possibilities related to our system which can make life simpler.

\section{PROBLEMS FACED}

Creating the dataset because we have no idea about how much data is required for the machine to achieve good accuracy and performance. Installation of dependencies because we are using a few libraries which belong to $\mathrm{C}++, \mathrm{C}$ background which are not compatible with machine learning platforms. Example: - dlib Graphical user interface, creating graphical user interface is not quite easier than requiring perfect measurement to create a perfect graphical user interface. Most important thing is to work with algorithms like canny detection, Hough transform, eye blink algorithm is complex to execute.

\section{CONCLUSION}

The study is providing promising results in ensuring the driver's safety, this system has three models the first model detects drowsiness in rapid manner and it is also capable of differentiating the eye blink and drowsiness which helps drivers moving in sleepy state. The system considers the eye to be opened or not if the eye is closed more than 0.3 seconds then the system gives an alert which ensures safety. Second model lane detection system, this system is implemented for detecting lanes and to reduce the accidents recorded in past few years. System alerts the driver when the driver is moving into the wrong lane, due to which the driver pays attention to it and will move in the proper lane. The third model vehicle maintenance prediction which predicts the maintenance of the system by considering the input given to the system. Driver safety systems can be implemented in vehicles to make sure that safety of driver is taken into consideration and this system has the probability of reducing accidents.

\section{REFERENCES}

[1] (Chirra, Uyyala, \& Kolli, 2019). Drowsiness detection using machine learning based on eye state.

[2] (Zahara, Miri Ashtiani, \& Mikaili, 2011). EEG based drowsiness detection system for driver safety using chaotic features and statistical analysis.

[3] (Ch. V. Ramireddy \& K. V. Krishna Kishore, 2013). Facial expression classification using kernel based PCA with fused DCT and GWT features. 
[4] (Eyosiyas Tadesse, Weihua Sheng, \& Meiqin Liu, 2014). Driver drowsiness detection through HMM based dynamic modeling.

[5] (Kwan Woo Lee, Hyo Sik Yoon, Jong Min Song, \& Kang Ryoung Park , 2018). Convolutional neural network-based classification of driver's emotion using multi camera sensors.

[6] (Rashmi N. Mahajan \& Dr. A. M. Patil, 2015). Lane departure warning system.

[7] (Sun, Tsai, \& Chan, 2006), HSI color model-based lanemarking detection.

[8] (Hui, Jean-Yves, \& Jean, 2010). General road detection from a single image.
[9] (Md. Rezwanul Haque, Md. Milon Islam, Kazi Saeed Alam, \& Hasib Iqbal, 2019). A computer vision-based lane detection approach.

[10] (R. Fisher, 2003). Hough's Transform

[11] (Gandhi, 2018). Support Vector Machine-Introduction to Machine Learning Algorithm.

[12] (R. Fisher, Canny Edge Detector, 2003). Canny Edge Detection.

[13] (Rosebrook, 2017). Eye Blink Detection with OpenCV, Python and dilb.

[14] (S. Perkins, 2003). Gaussian Smoothing.

[15] (Huang \& Yang-Wang, 2018). Applications of Support Vector Machine (SVM) Learning in Cancer Genomics 